Acta Crystallographica Section D

Biological

Crystallography

ISSN 0907-4449

Tjaart A. P. de Beer, ${ }^{\text {a }}$ Roman A. Laskowski, ${ }^{a}$ Mark-Eugene Duban, ${ }^{\text {b }}$ A. W. Edith Chan, Wayne F. Anderson ${ }^{b}$ and Janet M. Thornton ${ }^{\mathrm{a}}$ *

a European Molecular Biology Laboratory, European Bioinformatics Institute (EMBL-EBI), Wellcome Trust Genome Campus, Hinxton, Cambridge CB10 1SD, England, 'benter for Structural Genomics of Infectious Diseases, Northwestern University Feinberg School of Medicine, Chicago, Illinois, USA, and ${ }^{\mathrm{c}}$ Biological and Medicinal Chemistry Group, Wolfson Institute for Biomedical Research, University College London, London WC1E 6BT, England

Correspondence e-mail: thornton@ebi.ac.uk

\title{
LigSearch: a knowledge-based web server to identify likely ligands for a protein target
}

Identifying which ligands might bind to a protein before crystallization trials could provide a significant saving in time and resources. LigSearch, a web server aimed at predicting ligands that might bind to and stabilize a given protein, has been developed. Using a protein sequence and/or structure, the system searches against a variety of databases, combining available knowledge, and provides a clustered and ranked output of possible ligands. LigSearch can be accessed at http:// www.ebi.ac.uk/thornton-srv/databases/LigSearch.

\section{Introduction}

Over the last few years a number of public small-molecule databases have been established, each with its own focus. The main databases are KEGG (Kotera et al., 2012), BRENDA (Scheer et al., 2011), ChEMBL (Gaulton et al., 2012), ChEBI (de Matos et al., 2012), ZINC (Irwin et al., 2012) and PubChem (Bolton et al., 2008). The KEGG small-molecule database focuses on substrates and products found in metabolic pathways and contains about 17000 molecules. BRENDA is a collection of enzyme functional data and, as such, contains information about the small molecules $(\sim 175000)$ involved in enzymatic reactions. In comparison, the ChEMBL database contains $\sim 1.4$ million bioactive, drug-like small molecules as well as data relating to various molecular properties such as $\log \mathrm{P}$ and Lipinski parameters. ChEBI is an ontology-based dictionary of $\sim 34000$ biologically interesting small molecules. ZINC complements these databases by providing a free database of $\sim 21$ million commercially available small molecules. The PubChem database contains information on the biological activities of small molecules as derived from the various NIH databases and contains data on over 100 million substances. Finally, the Worldwide Protein Data Bank (wwPDB; Berman et al., 2003) holds over 64000 threedimensional structures of protein-ligand complexes and hence is an especially rich source of information on the binding of small molecules to proteins.

Given that a protein-ligand complex can provide valuable data on both the binding site of a protein and its biochemical function, crystallographers often need to identify small molecules that might bind to their protein. Rather than use trial and error, which is expensive and time-consuming, it is far better to identify potential ligands prior to the start of the experiments. However, even identifying likely molecules can involve a great deal of time and effort. For this reason, we have developed LigSearch, a web server that automates the process of identifying potential ligands for a given protein. The method uses sequence information and so is suitable for proteins of known and unknown three-dimensional structure alike.
Received 11 April 2013 Accepted 8 August 2013 
It should be noted that the aim of LigSearch is not to identify a protein's binding site, although in some cases this may be a spin-off. There are plenty of methods that do this already, some using structural information, some using sequence information and others using a combination of both. The server merely aims to use various existing resources to identify small molecules that are likely to bind to a given protein. It then clusters the results, grouping the molecules by their similarity, and ranks the clusters and the molecules within each cluster using a scoring scheme that aims to place the more promising hits nearer the top of the output.

\section{Methods}

The LigSearch pipeline is shown schematically in Fig. 1 and is described in more detail below. The user can submit a protein sequence (either via a UniProt ID or a pasted sequence) or a protein structure (via a PDB code or an uploaded PDB file), from which the sequence is extracted. Results are emailed to the user in the form of a password-protected link to a web page of ranked ligand hits.

\subsection{Sequence searches}

The submitted protein sequence is first searched against the curated entries in UniProtKB/Swiss-Prot (The UniProt Consortium, 2012) using BLAST (Altschul et al., 1990). The top 20 sequences matched represent the query sequence plus a set of its closest relatives. The UniProt identifiers for each of these 20 hits are searched against ChEMBL, ChEBI and KEGG, using their respective web services, to retrieve the small-molecule compounds that these databases have associated with each of the given proteins. For the most part, these data come from the scientific literature. ChEMBL search results are filtered to remove compounds having no binding constants and those with an $\mathrm{IC}_{50}$ value of more than $100 \mathrm{nM}$. ChEBI search results are filtered to exclude all compounds with fewer than four atoms and/or a molecular weight of less than $50 \mathrm{Da}$. The results from the three searches are combined and duplicates are removed, giving a list of compounds known to be associated with the query sequence and/or its closest relatives.

\subsection{Searches against the PDB}

The original sequence is then searched against the protein sequences in the PDB. To improve the chances of successful matches, and to increase the number of hits, the sequence is first broken up into regions that are likely to correspond to structural or sequence domains. This is performed as follows. The top $20 B L A S T$ hits found above are located in the Gene3D database (Lees et al., 2012), which primarily identifies likely structural domains in 15 million UniProt sequences. Gene3D is compiled by deriving hidden Markov models (HMMs) to map sequences to the protein structural domains defined in the CATH domain database (Sillitoe et al., 2013). Any long stretch of sequence that cannot be mapped to a CATH domain is instead mapped to a Pfam (Finn et al., 2010) sequence domain.

From the Gene3D domain assign-
Structure Uploaded file PDB code

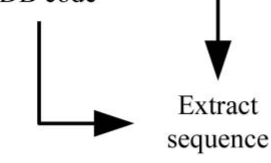

\section{Sequence}

Previous result ID$$
\text { UniProt ID }
$$
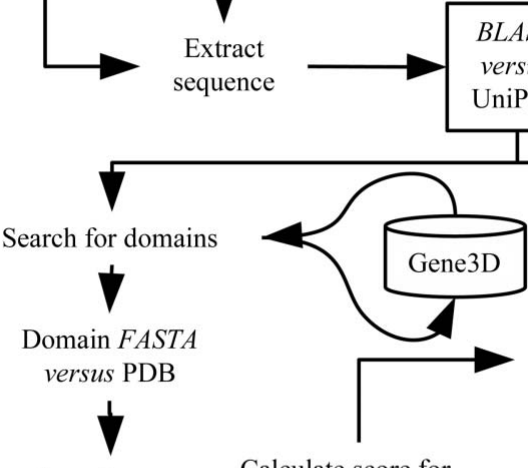

Superimpose structures

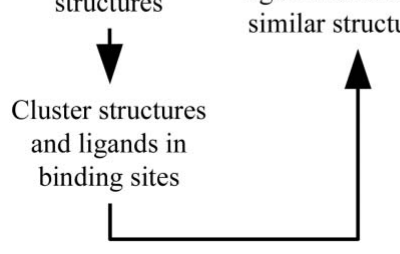

Calculate score for ligands binding to imilar structures

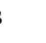

Pasted sequence

Search for domains

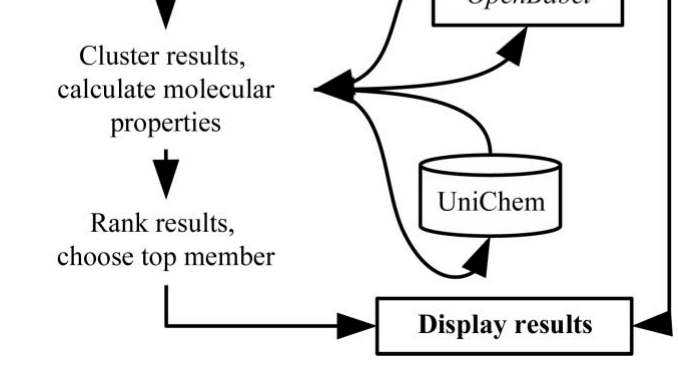

Figure 1

A schematic outline of the workflow in LigSearch. The user can submit either a structure or a sequence. ments for the 20 UniProt sequences, the query sequence is partitioned into predicted domains. Starting with the most similar sequence, the domains are applied to the query sequence provided that they do not overlap with a previously assigned domain. Once the domains have been assigned, any unassigned regions of over 100 residues are designated domains of unknown type. Linker sections between domains are split in half and each half is assigned to the domain adjacent to it.

The sequence of each of these protein domains is then searched against the sequences in the PDB. Again, to increase the chances of successful matches, the database of sequences contains not just the full-length sequences of each protein chain in the PDB but also the sequences corresponding to each CATH domain. The latter are particularly important for matching 'split' domains, which might otherwise be missed by the sequence search. For example, PDB entry 1got 
consists of two CATH domains, the first being a domain comprising residues 6-57 and 177-331 and thus 'split' over two segments of the protein, while the second domain spans the region in between, residues $58-176$.

The search itself is performed using FASTA (Lipman \& Pearson, 1985) and a multiple alignment is derived from the resultant pairwise sequence alignments. For each domain searched, the structure with the best match (based upon the maximum Smith-Waterman score produced by FASTA) is used as a reference structure and all others are superposed onto it using the main-chain atoms of equivalent residues in the alignment. Several iterations of superposition may be required to obtain an r.m.s.d. below a $12 \AA$ cutoff, with the residues having the highest r.m.s.d. values being removed at each iteration. This compensates for any imperfections in the sequence alignment.

The superposition of all of the structures onto the reference structure brings with it any bound ligands. The net result is that the various ligands overlap in one or more binding sites of the superposed proteins. These ligands represent the predicted binding partners of the query protein. A scoring system is used to order them from the most to the least promising candidates. The scores take into account the numbers and types of interaction that each ligand makes with its protein partner and also the similarity between the residues that it interacts with and the corresponding residues in the query protein. Specifically, the ligand scores 1 for each hydrogen bond it makes to the protein, with the score being 2 if the interaction is with a similar residue to that in the query sequence, 3 if it is with an identical residue type and -1 if there is no equivalent (i.e a gap in the alignment). The equivalent scores for nonbonded contacts are $0,1,2$ and -1 , respectively, although where there are several contacts to any given protein residue only one is counted.

The scoring system is somewhat arbitrary and is difficult to optimize without extensive experimental ligand-binding data; however, its aim is merely to provide a qualitative ranking of ligands according to how similar the residues they interact with are to the corresponding residues in the query sequence.

Many ligands in the PDB interact with more than one protein domain, so a domain-based sequence search and superposition will miss any interactions that the ligand makes with other domains. This is taken into account by merging the results from the separate domain searches.

\subsection{Molecular similarity and result ranking}

The ligands identified by the three sequence searches and those from the search against the PDB are clustered according to their molecular similarity as calculated by SMSD (Small Molecule Subgraph Detector; Rahman et al., 2009). SMSD computes the maximum common subgraph between two small molecules and provides a similarity score based on the matching subgraphs. Clustering uses a similarity cutoff of 0.4 between the most distant members of the cluster and is solely based on molecular similarity and not on whether the molecules bind in the same protein binding site.
The clusters obtained are ranked on the basis of the highest interaction score for the PDB ligands in each cluster. Within each cluster the PDB ligands are ranked by their interaction scores, while the hits from ChEMBL, ChEBI and KEGG, having no interaction score, are listed at the end of each cluster in decreasing order of the sequence similarity of their associated protein to the query sequence and then by their number of cross-references in UniChem (Chambers et al., 2013). UniChem is a nonredundant database of links between chemical structures and EMBL-EBI chemistry resources. The number of cross-references indicates in how many other databases the small molecule appears. This provides a qualitative measure of its likely 'importance'.

OpenBabel (O'Boyle et al., 2011) is used to calculate various molecular properties such as $\log \mathrm{P}$ and the polar surface area where such data are lacking. The ordered list of predicted ligands can be downloaded in a tab-separated file.

\subsection{Validation and benchmarking}

To validate the LigSearch pipeline, we chose a set of enzymes as our test group of proteins. For the most part, the molecules that bind to enzymes are known (the reactants, products and any cofactors) and hence provide a means of validating the LigSearch predictions. However, because of the very fact that the cognate ligands are known, they will inevitably be returned by the KEGG, ChEBI and ChEMBL searches and so such hits need to be discarded before analysing what is left.

Furthermore, if the three-dimensional structure of any of the enzymes is known, this will bias the results returned from the searches against the PDB. To prevent this, we first used the Enzyme Structures Database, part of PDBsum (Laskowski, 2009), to select a data set of enzymes which have no threedimensional structure in the PDB. This gave 3431 EC classes (as of July 2013). From these, we selected only those enzymes whose cognate molecules were given in the ENZYME database (Bairoch, 2000) and had an associated .mol file in the Enzyme Structures Database. This was to ensure that the correct answers (i.e the substrates and/or cofactors) were known and could be compared against the molecules returned by LigSearch. The result was a set of 2334 enzymes.

A list of proteins belonging to each of these enzyme classes was then extracted from UniProtKB/Swiss-Prot (i.e. the reviewed part of UniProtKB). The 2334 enzyme classes encompassed 195754 UniProtKB sequences. To select a manageable data set, the sequences were randomly selected from this list, ensuring that no EC class was represented more than once, to give 200 proteins. The number of identifiable reaction molecules associated with this set was 620 . The search sequences and reaction molecules are listed in Supplementary Table S1, ${ }^{1}$ together with the results of the searches, as described below.

\footnotetext{
${ }^{1}$ Supplementary material has been deposited in the IUCr electronic archive (Reference: RR5044). Services for accessing this material are described at the back of the journal.
} 
Firstly, we analyzed the results returned by the searches against the PDB. For each of the 200 protein sequences, the PDB ligands returned by LigSearch were compared against the protein's cognate molecules using the SMSD program. For $102(51 \%)$ of the proteins at least one of the molecules suggested by LigSearch was a perfect match to one of these cognate molecules. In a further 18 cases a molecule with a match score of 0.8 or higher to a known substrate was identified. Thus, for 120 of the 200 enzymes $(60 \%)$ an identical or very similar molecule to a known binder was identified in the PDB (see Fig. 2a). These were not merely trivial matches from close homologues. Just two of the 200 came from a protein with sequence identity higher than $65 \%$ to the query sequence. Over $50 \%$ came from proteins with a sequence identity of $30 \%$ or less (see Fig. $2 b$ ).

As described above, the LigSearch score for each PDB hit reflects the similarity between the residues interacting with the ligand in the PDB complex and the corresponding residues in the query protein. The higher the score, the more equivalent interactions with identical or similar residue types are possible. Indeed, the best matches in the enzyme data set tend to have the highest LigSearch scores (Fig. 2c), so the scores do provide a guide to which molecules are more likely to bind to the query protein. Indeed, from the results in Fig. 2(c) it would appear that ligand scores higher than around 10-15 tend to be associated with the correct answers.

Secondly, to test the results returned by the ChEBI, ChEMBL and KEGG searches, we took each cognate molecule in turn and counted how many other molecules were returned in the same cluster as the cognate molecule. Fig. $2(d)$ shows the results. For $22(11 \%)$ of the enzymes none of the molecules returned by the ChEBI, ChEMBL and KEGG searches were similar enough to any of the cognate molecules to be in the same cluster. However, for the remaining 178 $(89 \%)$ of the enzymes at least one of the small molecules returned was similar to one of the cognate ligands.

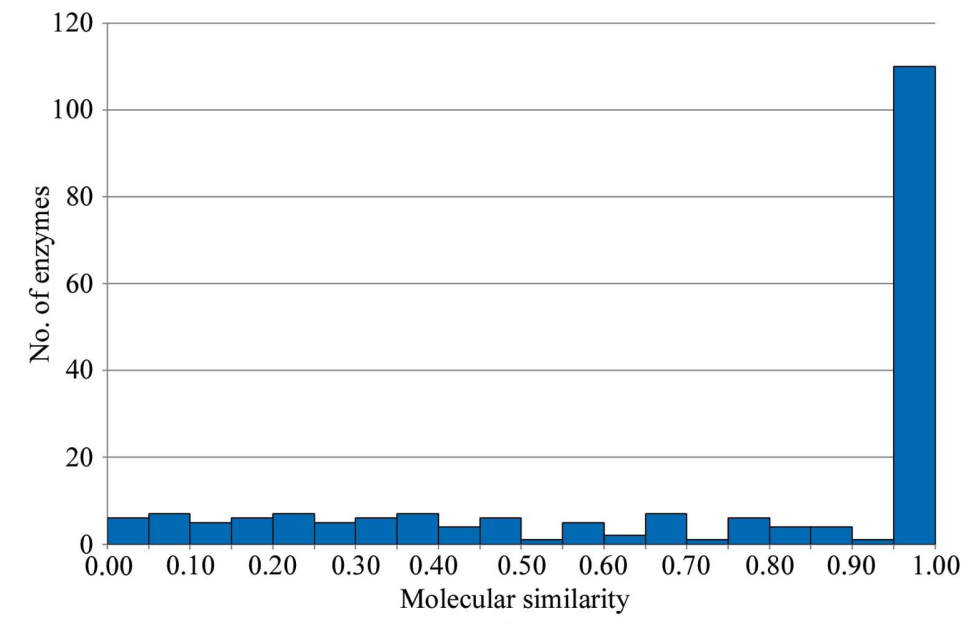

(a)

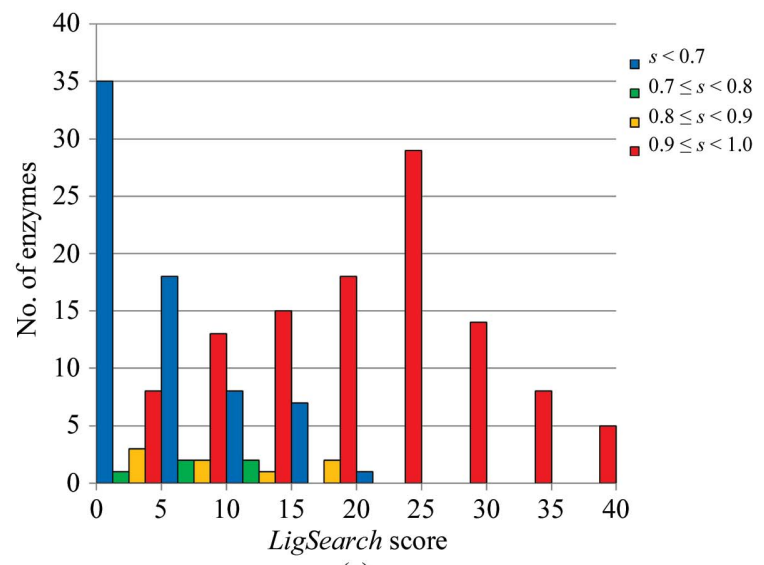

(c)

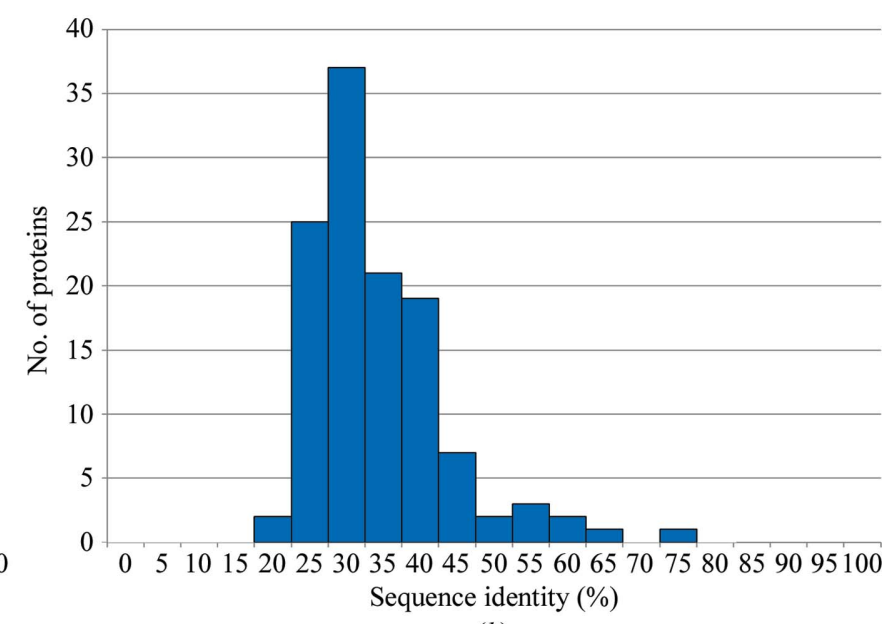

(b)

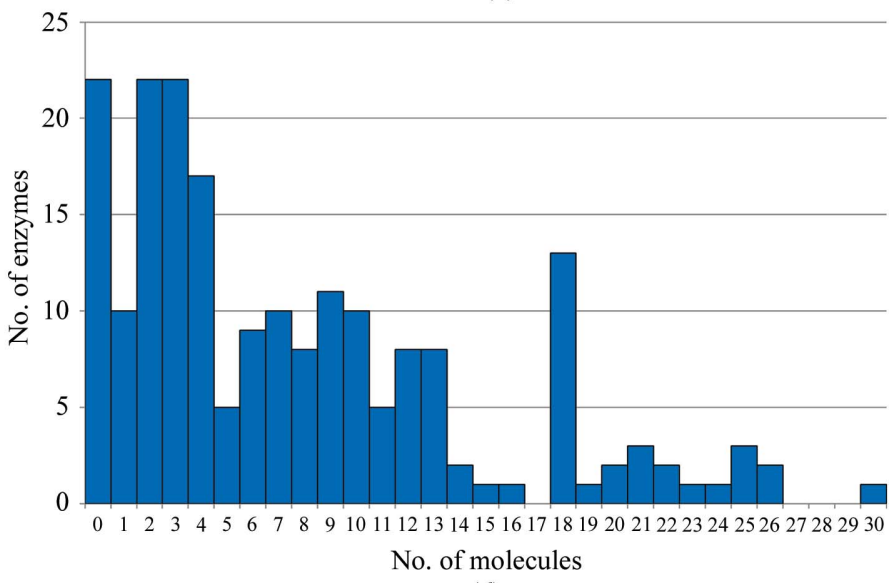

(d)

\section{Figure 2}

Validation results for LigSearch runs on 200 randomly selected enzymes with no three-dimensional structural model in the PDB. (a) Histogram of the molecular-similarity scores for the closest PDB ligand match, as computed by the SMSD program, to any of the enzyme's cognate ligands. (b) Histogram of the sequence identities between the query enzyme sequence and the PDB protein from which the best ligand match has a similarity of 0.8 or greater to one of the cognate ligands. (c) Histogram of the LigSearch scores for the best matches to cognate ligands. The counts are grouped into four sets according to the similarity score, $s$, of the best-matching molecule. Lowest similarity scores $(s<0.7)$ are shown in blue, scores $0.7 \leq s<0.8$ are shown in green, scores $0.8 \leq s<0.9$ are shown in orange and closest matches with $s \geq 0.9$ are shown in red. (d) Histogram of counts of molecules with similarity $s \geq 0.8$ to at least one of the enzyme's cognate ligands as returned by LigSearch for the non-PDB hits. The cognate molecules themselves are, of course, excluded from the results. 
Table 1

The 26 molecules manually selected in February 2012 for crystallization trials of $S$. aureus thymidylate synthase (UniProt ID P65248) and used here for testing the LigSearch results.

The molecules that were trialled are shown in bold and the two from which crystal structures were obtained are annotated with footnotes. The molecules have been grouped here by the three LigSearch clusters that they occurred in: LigSearch clusters 1, 3 and 15. The rightmost columns show details of the PDB entry from which the LigSearch match came.

\begin{tabular}{|c|c|c|c|c|}
\hline Candidate compound & LigSearch & $\begin{array}{l}\text { PDB } \\
\text { code } \\
\text { (chain) }\end{array}$ & Score & $\begin{array}{l}\text { Sequence } \\
\text { identity } \\
(\%)\end{array}$ \\
\hline \multicolumn{5}{|l|}{ LigSearch cluster 1} \\
\hline $\begin{array}{l}P^{1} \text {-(5'-Adenosyl)- } P^{5} \text {-(5'-thymidyl)pentaphosphate } \\
\quad \text { (Fig. } 4 a)\end{array}$ & T5A & $4 \operatorname{tmk}(A)$ & $29 / 33$ & 34.0 \\
\hline $\begin{array}{l}P^{1} \text {-(5'-Adenosyl) }-P^{5}-\left[5^{\prime}-\left(3^{\prime} \text {-azido- } 3^{\prime} \text {-deoxythymidyl }\right)\right] \\
\text { pentaphosphate }\end{array}$ & Z5A & $5 \operatorname{tmp}(A)$ & $25 / 29$ & 34.0 \\
\hline Phosphoaminophosphonic acid adenylate ester & ANP & 1nmy $(A)$ & $23 / 30$ & 22.6 \\
\hline Adenosine 5'-diphosphate & ADP & $2 \operatorname{cdn}(A)$ & $23 / 32$ & 24.3 \\
\hline Adenosine $5^{\prime}$-triphosphate & ATP & $1 \mathrm{e} 2 \mathrm{q}(A)$ & $18 / 27$ & 22.6 \\
\hline $2^{\prime}$-Deoxyguanosine $5^{\prime}$-triphosphate & DGT & $2 \mathrm{vp} 2(A)$ & $18 / 38$ & 26.2 \\
\hline Guanosine 5'-monophosphate & $5 \mathrm{GP}$ & $1 \mathrm{ex} 7$ & $7 /-$ & 23.7 \\
\hline \multicolumn{5}{|l|}{ LigSearch cluster 3} \\
\hline Thymidine $5^{\prime}$-diphosphate (Fig. 4b) & TYD & 3hjn $(A)$ & $24 / 27$ & 41.8 \\
\hline Thymidine $5^{\prime}$-phosphate $\dagger$ & TMP & $2 \operatorname{ccg}(A)$ & $21 / 21$ & 100.0 \\
\hline Thymidine $5^{\prime}$-triphosphate & ТTP & $2 \mathrm{vp} 0(A)$ & $21 / 37$ & 26.2 \\
\hline 2'-Deoxycytidine $5^{\prime}$-triphosphate & $\mathrm{DCP}$ & $2 \mathrm{vp} 4(A)$ & $20 / 34$ & 26.2 \\
\hline Thymidine & THM & $4 \operatorname{esh}(A)$ & $13 / 15$ & 35.9 \\
\hline $3^{\prime}$-Deoxythymidine $5^{\prime}$-monophosphate & 2DT & $1 \mathrm{nn} 0(A)$ & $11 / 14$ & 22.6 \\
\hline $3^{\prime}$-Azido-3'-deoxythymidine $5^{\prime}$-monophosphate $\ddagger$ & ATM & $1 \mathrm{e} 99(A)$ & $11 / 19$ & 22.6 \\
\hline $3^{\prime}$-Fluoro-3'-deoxythymidine monophosphate & FDM & $1 \mathrm{nmx}(A)$ & $11 / 14$ & 22.6 \\
\hline 2'-Deoxycytidine & $\mathrm{DCZ}$ & $1 \mathrm{j} 90(A)$ & $10 / 22$ & 22.7 \\
\hline (E)-5-(2-Bromovinyl)-2'-deoxyuridine $5^{\prime}$-monophosphate & BVP & 2w0s $(A)$ & $8 / 12$ & 25.3 \\
\hline 5-Hydroxymethyluridine $2^{\prime}$-deoxy-5'-monophosphate & $5 \mathrm{HU}$ & $1 \mathrm{mrs}(A)$ & $8 / 15$ & 28.6 \\
\hline 3'-Deoxy-3'-aminothymidine monophosphate & NYM & $1 \mathrm{nmz}(A)$ & $8 / 15$ & 22.6 \\
\hline $2^{\prime}, 3^{\prime}$-Dideoxycytidine $5^{\prime}$-monophosphate & DOC & $2 \operatorname{vp} 9(A)$ & $6 / 20$ & 25.8 \\
\hline 5-Bromovinyldeoxyuridine & BVD & 2vqs $(A)$ & $5 / 11$ & 26.8 \\
\hline Gemcitabine & GEO & $2 \operatorname{vpp}(A)$ & $5 / 17$ & 26.7 \\
\hline 5-Fluorouridine $5^{\prime}$-monophosphate & $5 \mathrm{FU}$ & 2vp6 $(A)$ & $5 / 19$ & 25.8 \\
\hline \multicolumn{5}{|l|}{ LigSearch cluster 15} \\
\hline $\begin{array}{l}\text { 4-(2-Hydroxyethyl)-1-piperazineethanesulfonic acid } \\
\text { (Fig. } 4 c \text { ) }\end{array}$ & EPE & $2 \operatorname{plr}(A)$ & $6 / 9$ & 28.3 \\
\hline \multicolumn{5}{|l|}{ Not found by LigSearch } \\
\hline $3^{\prime}$-Azido-3'-deoxythymidine & - & - & - & - \\
\hline Diphosphate & - & - & - & - \\
\hline
\end{tabular}

$\dagger$ Structure solved as PDB entry $4 \mathrm{dwj} . \quad$ 末 Structure solved as PDB entry 4eaq.

Together, the validation study suggests that in the majority of cases the answers returned by LigSearch include molecules that are highly likely to bind, owing to their high similarity to known binders, and these molecules tend to be those that score highly using the LigSearch interaction score.

\section{Results}

To demonstrate the usefulness of the system in practice, we obtained ligand-testing data from one of the crystallographers at the Midwest Center for Structural Genomics (MCSG). Her project had necessitated a search for candidate molecules to cocrystallize with thymidylate synthase from Staphylococcus aureus (UniProt ID P65248). Using results from literature mining, she had identified a number of potential compounds in February 2012. Of these, 26 were selected and 13 were used in crystallization trials (E. Filippova, personal communication). Table 1 lists the 26 compounds. The structures of two proteinligand complexes were eventually solved and deposited in the
PDB as entries 4dwj and 4eaq in February and March 2012, respectively. In fact, $4 \mathrm{dwj}$ was a trivial case as the ligand selected had already been solved in complex with the same protein (PDB entries $2 \mathrm{ccg}$ and $2 \mathrm{ccj}$ ).

We submitted this protein sequence to LigSearch to compare the hits returned against the molecules that had been manually compiled. The PDB contains many thymidylate synthase structures from various organisms, so it was not surprising that LigSearch returned many hits. All structures solved after February 2012 were discarded in order to present the results as they would have been at the time of the original study. In all, 62 unique ligands were matched in the PDB. Additionally, a further 126 unique molecules were obtained from ChEBI. In this example, no hits were returned by the ChEMBL and KEGG searches.

The clustering of all of the candidate molecules by $S M S D$ resulted in 47 separate clusters, five of which contained a single metal ion. Fig. 3 shows the highest-scoring members of each of these 47 clusters plotted using multi-dimensional scaling on the basis of their all-by-all similarities.

The top-scoring clusters, ranked 1, 2 etc., tend to group in the bottom righthand corner of the plot. The metals and various very small molecules are grouped at the bottom left.

Most of the 26 compounds from the manual selection exercise listed in Table 1 were identified in the LigSearch output and indeed fell into three of LigSearch's clusters, 1,3 and 15 , as shown in the table. The top-scoring ligand in each of these three clusters is depicted in Fig. 4, showing the atoms that interact with the protein. In fact, LigSearch identified even higher scoring molecules than those that had been selected by hand, but these are not shown in the table. LigSearch missed two of the 26 manually selected compounds, but in both cases the compounds are substructures of other molecules returned by LigSearch and thus in effect are not significant omissions.

The rightmost columns of the table show the PDB entries from which the ligands came and the sequence similarity of each protein to the query sequence. Many of the latter lie in the $20-30 \%$ range, suggesting the predicted ligands come from distantly related proteins. Their high interaction scores, however, are suggestive of conservation in the binding site and indicate that there is a strong chance the ligands may bind to the query protein. 
Fig. 5 shows an example of such a case. It compares the protein-ligand interactions for the same $3^{\prime}$-azido- $3^{\prime}$-deoxythymidine $5^{\prime}$-monophosphate ligand bound to $S$. aureus thymidylate synthase in PDB entry 4eaq and human thymidylate synthase in PDB entry 1e99. Despite the low overall sequence identity between the two proteins $(22.6 \%)$, there are several conserved residues in the binding site that make identical interactions (three arginines and one phenylalanine) and other interactions made by similar residues in the same three-dimensional locations in the two structures. Cases such as this demonstrate that matches to even distantly related proteins can provide valid predictions about which ligands are worth considering.

\section{Discussion}

LigSearch is a convenient tool for identifying possible ligands for a given protein and hence can provide crystallographers with a list of candidate molecules for crystallization trials. It reduces the amount of manual searching and literature mining by providing the results automatically and conveniently clustering the resultant molecules into groups according to molecular similarity.

Arguably, the most useful matches are those that come from protein-ligand complexes in the PDB. Even if the proteins are distant relatives, the match can identify likely binding residues and indicate where the ligand might bind. The clusters represent a set of molecules that are all at least $40 \%$ similar to each other. This does not imply that they bind in the same binding site in a protein, as the clustering is performed based on molecular similarity and not three-dimensional location.

These clusters provide a very good way to identify any potential molecular frameworks that might bind to the protein. In addition, the ChEBI and ChEMBL results provide a good enrichment of the main framework in each cluster. In this example, one of the clusters has thymidine $5^{\prime}$-diphosphate as the highest scoring compound. From the ChEBI and ChEMBL results, another six variants such as $5^{\prime}$-thymidilic acid and thymidine triphosphate were added.

When looking at all of the clusters found for our example, it becomes clear that there are a large number of different molecular frameworks present. The highest scoring clusters

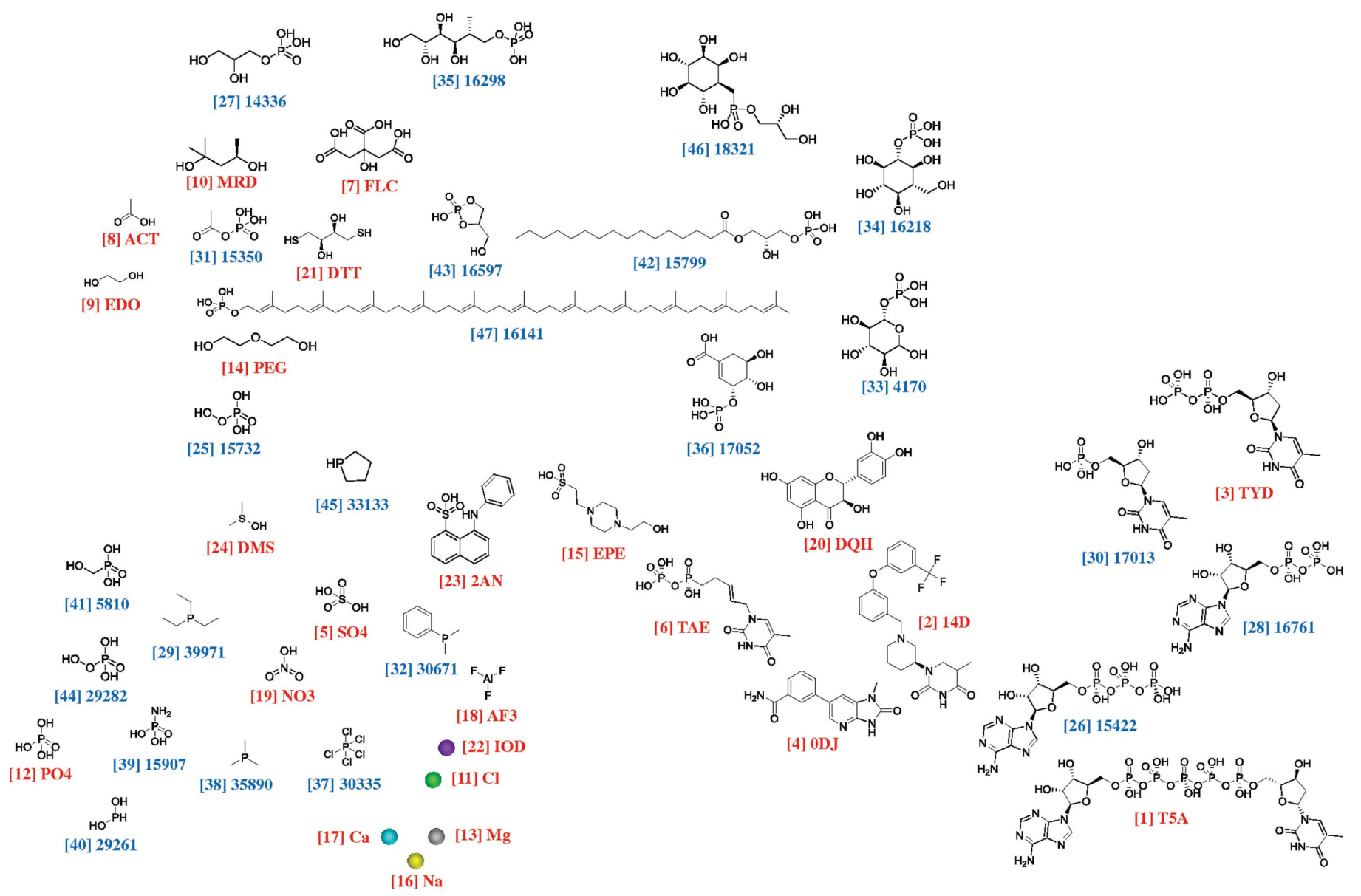

Figure 3

A plot of the top-scoring molecules in each of the 47 clusters returned by LigSearch for UniProt entry P65248. The molecules have been laid out using multi-dimensional scaling on the basis of their all-by-all similarities. Thus, similar molecules tend to be grouped together. The labels show the cluster number in square brackets and the PDB Het Group three-character name or ChEBI identifier. Red labels correspond to molecules from matches to PDB entries, while blue labels are molecules returned by ChEBI searches. The molecular diagrams were plotted using ChemDraw (http:// www.cambridgesoft.com). 


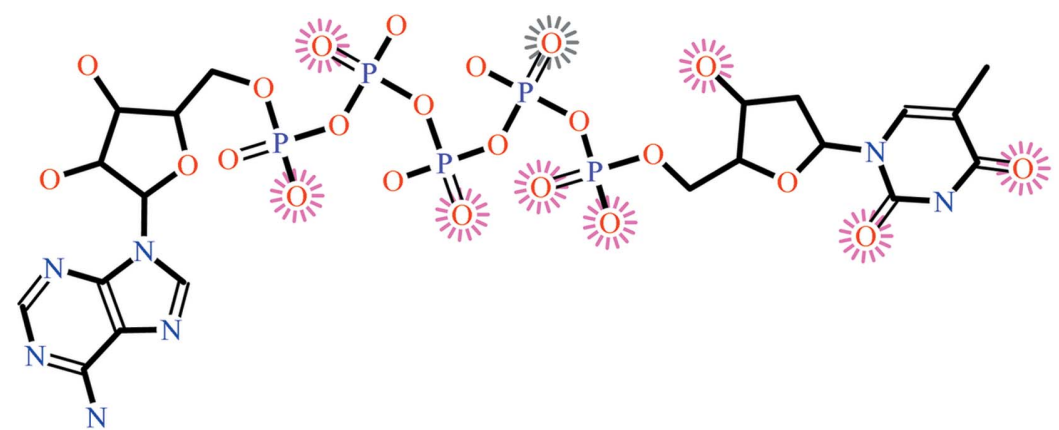

$(a)$

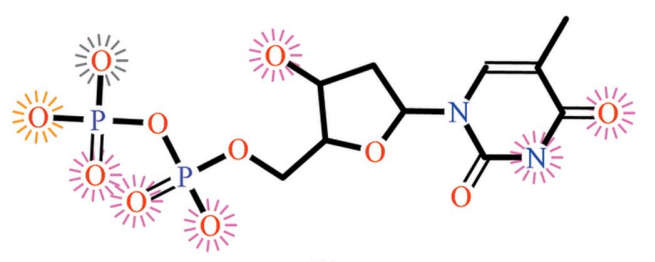

(b)

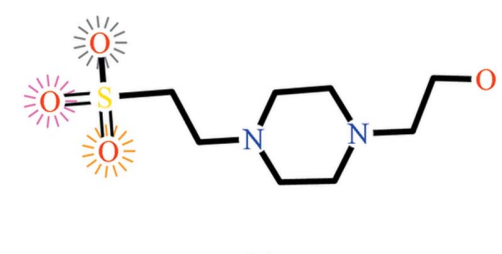

(c)
Figure 4

Three cluster representatives for the molecules listed in Table 1. The molecules are annotated according to the interactions that they make with the protein in the top-scoring PDB entry for the cluster. Atoms making hydrogen bonds to protein are depicted with spokes radiating from them, while hydrophobic interactions have a grey circle around them (none in this example). The colour of the spokes corresponds to the similarity of the residue to which the hydrogen bond is made and the corresponding residue in the query protein (which in this case is thymidylate synthase from S. aureus; UniProt ID P65248): red for identical residue type, orange for similar and dark grey for different. The images are provided in the results section for every query with PDB hits. The molecules and the PDB entries from which the data come are $(a) P^{1}$ - $\left(5^{\prime}\right.$-adenosyl $)-P^{5}-\left(5^{\prime}\right.$ thymidyl)pentaphosphate (PDB entry 4tmk), (b) thymidine $5^{\prime}$-diphosphate (PDB entry 3 hjn) and (c) 4-(2-hydroxyethyl)-1-piperazineethanesulfonic acid (PDB entry 2pir).

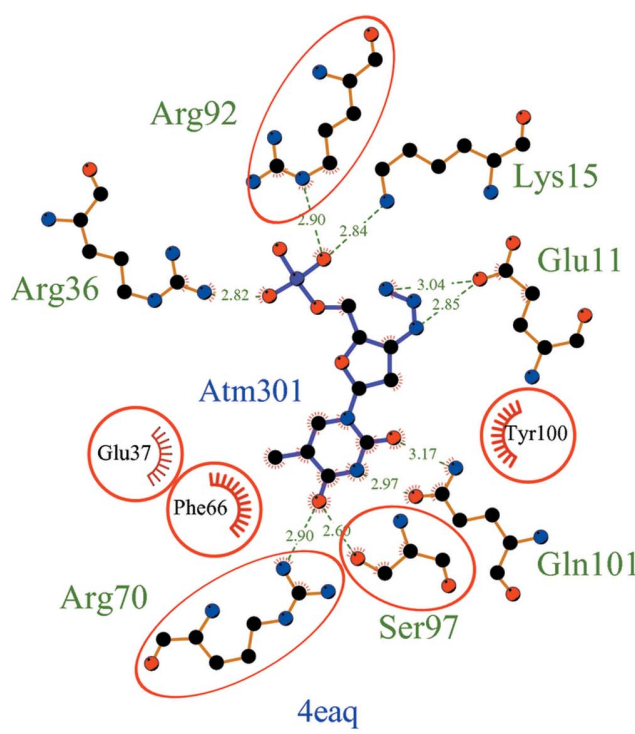

(a)

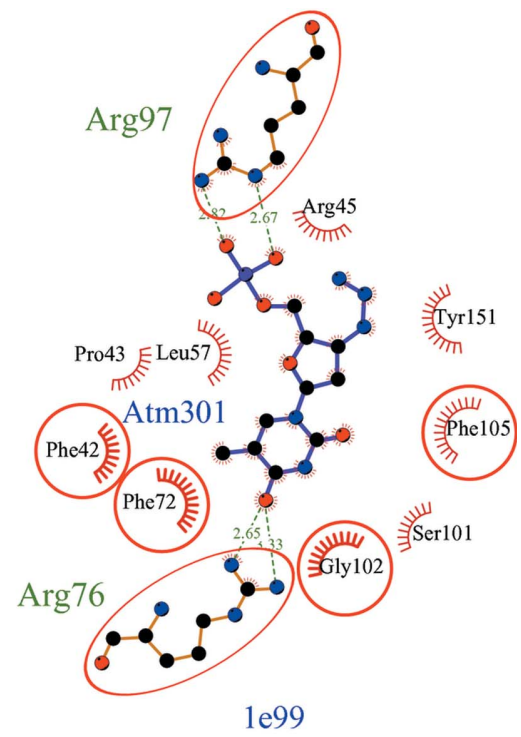

(b)
Figure 5

A schematic diagram of the protein-ligand interactions in two distantly related proteins: $(a)$ thymidylate synthase from $S$. aureus (PDB entry 4eaq) and (b) human thymidylate synthase (PDB entry 1e99). The ligands (blue bonds) in both are identical: $3^{\prime}$-azido-3'-deoxythymidine $5^{\prime}$-monophosphate. Equivalent protein residues in the two plots are circled in red and occupy the same positions in each plot: for example, Glu37 is equivalent to Phe42, Phe66 is equivalent to Phe72 and Tyr100 is equivalent to Phe105. Hydrogen bonds are depicted by green dotted lines and labelled with their length in $\AA$, while hydrophobic interactions are represented by red arcs whose spokes radiate towards the ligand. The diagram was generated using $\mathrm{LigPlot}^{+}$(Laskowski \& Swindells, 2011). tend to contain the substrate/product molecules as well as various versions of these molecules. A number of clusters consist of molecules commonly found in crystallization solutions, such as sulfate, phosphate and acetic acid. These molecules are difficult to exclude as they might be involved in the protein function. Some of the clusters contain highly reactive/unstable molecules such as phosphorus pentachloride and $3 \mathrm{H}$ phosphole. Owing to the nature of the ChEBI and ChEMBL databases and their annotation, these molecules will be included in the results but will usually cluster together. This makes it easier for the crystallographer to disregard them.

The ordering of the PDB ligand hits within each cluster relies on a somewhat arbitrary scoring scheme. The rationale for the weights assigned to each interaction seems reasonable, although it would require a great deal of experimental testing to try to optimize it. Possibly it cannot, and maybe it need not, be optimized. Larger ligands tend to give a better score as they usually make more interactions with the protein, but this in itself may be suggestive of a good candidate for binding to the query protein. We welcome any collaborations willing to help with further experimental testing and ranking improvements.

Some of the searches return results for highly volatile or unstable compounds as well as compounds known to be insoluble in water. Hence, one of the improvements that is planned for the future is a more chemistry-aware filter. The implemention of a smart rulebased system, in combination with other parameters such as $\log \mathrm{P}$, would improve the results by removing molecules that are unlikely to be of any practical or biological use. An additional improvement, currently in the planning stages, is that all hits found should be screened against the ZINC database, checking whether the compound is purchasable.

Another potential use for LigSearch might be to tackle the 'unknown ligand problem' in which a protein structure solved by X-ray crystallography is found to have mystery density belonging to some unknown molecule in the binding site. By submitting the sequence and/or 
structure to the LigSearch server, the homology searches might provide clues to the identity of the ligand.

This work was supported in part by National Institutes of Health grant GM094585, by the US Department of Energy, Office of Biological and Environmental Research under contract DE-AC02-06CH11357 (Midwest Center for Structural Genomics) and in part by Federal funds from the National Institute of Allergy and Infectious Diseases, National Institutes of Health, Department of Health and Human Services under Contract Nos. HHSN272200700058C and HHSN272201200026C. We would also like to thank EMBLEBI for providing the computational resources and support for TAPdB. Thanks to E. Filippova for providing a manually selected set of compounds for use in testing LigSearch.

\section{References}

Altschul, S. F., Gish, W., Miller, W., Myers, E. W. \& Lipman, D. J. (1990). J. Mol. Biol. 215, 403-410.

Bairoch, A. (2000). Nucleic Acids Res. 28, 304-305.

Berman, H., Henrick, K. \& Nakamura, H. (2003). Nature Struct. Biol. 10, 980.

Bolton, E., Wang, Y., Thiessen, P. A. \& Bryant, S. H. (2008). Annu. Rep. Comput. Chem. 4, 217-241.

Chambers, J., Davies, M., Gaulton, A., Hersey, A., Velankar, S., Petryszak, R., Hastings, J., Bellis, L., McGlinchey, S. \& Overington, J. P. (2013). J. Cheminform. 5, 3.
Finn, R. D., Mistry, J., Tate, J., Coggill, P., Heger, A., Pollington, J. E., Gavin, O. L., Gunasekaran, P., Ceric, G., Forslund, K., Holm, L., Sonnhammer, E. L. L., Eddy, S. R. \& Bateman, A. (2010). Nucleic Acids Res. 38, D211-D222.

Gaulton, A., Bellis, L. J., Bento, A. P., Chambers, J., Davies, M., Hersey, A., Light, Y., McGlinchey, S., Michalovich, D., Al-Lazikani, B. \& Overington, J. P. (2012). Nucleic Acids Res. 40, D1100-D1107.

Irwin, J. J., Sterling, T., Mysinger, M. M., Bolstad, E. S. \& Coleman, R. G. (2012). J. Chem. Inf. Model. 52, 1757-1768.

Kotera, M., Hirakawa, M., Tokimatsu, T., Goto, S. \& Kanehisa, M. (2012). Methods Mol. Biol. 802, 19-39.

Laskowski, R. A. (2009). Nucleic Acids Res. 37, D355-D359.

Laskowski, R. A. \& Swindells, M. B. (2011). J. Chem. Inf. Model. 51, 2778-2786.

Lees, J., Yeats, C., Perkins, J., Sillitoe, I., Rentzsch, R., Dessailly, B. H. \& Orengo, C. (2012). Nucleic Acids Res. 40, D465-D471.

Lipman, D. J. \& Pearson, W. R. (1985). Science, 227, 1435-1441.

Matos, P. de, Adams, N., Hastings, J., Moreno, P. \& Steinbeck, C. (2012). Methods Mol. Biol. 803, 273-296.

O'Boyle, N. M., Banck, M., James, C. A., Morley, C., Vandermeersch, T. \& Hutchison, G. R. (2011). J. Cheminform. 3, 33.

Rahman, S. A., Bashton, M., Holliday, G. L., Schrader, R. \& Thornton, J. M. (2009). J. Cheminform. 1, 12.

Scheer, M., Grote, A., Chang, A., Schomburg, I., Munaretto, C., Rother, M., Söhngen, C., Stelzer, M., Thiele, J. \& Schomburg, D. (2011). Nucleic Acids Res. 39, D670-D676.

Sillitoe, I., Cuff, A. L., Dessailly, B. H., Dawson, N. L., Furnham, N., Lee, D., Lees, J. G., Lewis, T. E., Studer, R. A., Rentzsch, R., Yeats, C., Thornton, J. M. \& Orengo, C. A. (2013). Nucleic Acids Res. 41, D490-D498.

The UniProt Consortium (2012). Nucleic Acids Res. 40, D71-D75. 